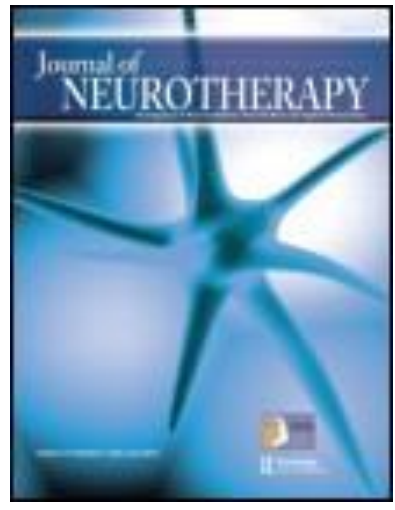

Journal of Neurotherapy: Investigations in Neuromodulation, Neurofeedback and Applied Neuroscience

\title{
EEG Biofeedback Training and Attention- Deficit/Hyperactivity Disorder in an Elementary School Setting
}

\author{
Dennis P. Carmody $\mathrm{PhD}^{\mathrm{a}}$, Diane C. Radvanski BS ${ }^{\mathrm{b}}$, Sonia Wadhwani BS ${ }^{\mathrm{C}}$ \\ Mary Jo Sabo $\mathrm{PhD}^{\mathrm{d}}$ \& Linda Vergara $\mathrm{MS}^{\mathrm{e}}$ \\ a Psychology Department, Saint Peter's College, Jersey City, NJ, USA \\ b Saint Peter's College, USA \\ C Fairleigh Dickinson University, USA \\ d Biofeedback Center, Suffern, New York, USA \\ e Enrico Fermi School, USA Published online: 20 \\ Oct 2008.
}

To cite this article: Dennis P. Carmody PhD , Diane C. Radvanski BS, Sonia Wadhwani BS, Mary Jo Sabo PhD \& Linda Vergara MS (2000) EEG Biofeedback Training and Attention-Deficit/Hyperactivity Disorder in an Elementary School Setting, Journal of Neurotherapy: Investigations in Neuromodulation, Neurofeedback and Applied Neuroscience, 4:3, 5-27, DOI: 10.1300/J184v04n03_02

To link to this article: http://dx.doi.org/10.1300/J184v04n03_02

\section{PLEASE SCROLL DOWN FOR ARTICLE}

(C) International Society for Neurofeedback and Research (ISNR), all rights reserved. This article (the "Article") may be accessed online from ISNR at no charge. The Article may be viewed online, stored in electronic or physical form, or archived for research, teaching, and private study purposes. The Article may be archived in public libraries or university libraries at the direction of said public library or university library. Any other reproduction of the Article for redistribution, sale, resale, loan, sublicensing, systematic supply, or other distribution, including both physical and electronic reproduction for such purposes, is expressly forbidden. Preparing or reproducing derivative works of this article is expressly forbidden. ISNR makes no representation or warranty as to the accuracy or completeness of any content in the Article. From 1995 to 2013 the Journal of Neurotherapy was the official publication of ISNR (WwW. Isnr.org); on April 27, 2016 ISNR acquired the journal from Taylor \& Francis Group, LLC. In 2014, ISNR established its official open-access journal NeuroRegulation (ISSN: 2373-0587; www.neuroregulation.org).

\section{THIS OPEN-ACCESS CONTENT MADE POSSIBLE BY THESE GENEROUS SPONSORS}

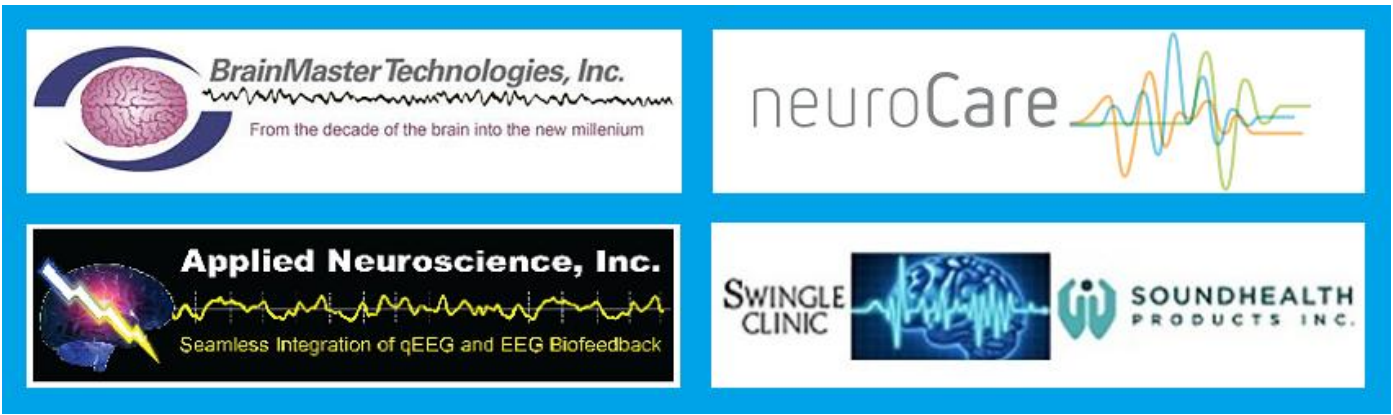




\title{
EEG Biofeedback Training and Attention-Deficit/Hyperactivity Disorder in an Elementary School Setting
}

\author{
Dennis P. Carmody, PhD \\ Diane C. Radvanski, BS \\ Sonia Wadhwani, BS \\ Mary Jo Sabo, PhD \\ Linda Vergara, MS
}

Dennis P. Carmody is Professor and former Chairperson of the Psychology Department at Saint Peter's College in Jersey City, NJ. His recent research evaluates the outcomes of interventions with children who have attentional deficits.

Diane C. Radvanski graduated summa cum laude and class valedictorian from Saint Peter's College. She worked as a technician for Biofeedback Consultants, Inc., on site conducting biofeedback training at an elementary school in Yonkers, NY.

Sonia Wadhwani received her Bachelors of Science degree from Saint Peter's College and is pursuing her graduate degree in psychology at Fairleigh Dickinson University.

Mary Jo Sabo is Administrator for the Biofeedback Center in Suffern, New York and administrates the EEG Biofeedback Program in three elementary public schools in Yonkers, NY.

Linda Vergara is Principal of the Enrico Fermi School for the Performing Arts. In 1995, she initiated and implemented the first biofeedback program in the public school system in Yonkers, NY.

Address correspondence to: Dennis P. Carmody, Saint Peter's College, Department of Psychology, 2641 Kennedy Boulevard, Jersey City, NJ 07306 (E-mail: carmody_d @spc.edu). 
ABSTRACT. Introduction: EEG biofeedback was conducted on site in an elementary school.

Method: An experimental group of eight children ages 8-10 completed 35-47 sessions of EEG biofeedback training over a six-month period. Four participants in the experimental group were diagnosed with Attention-Deficit/Hyperactivity Disorder (ADHD) and four were not diagnosed with ADHD. Eight children in the waitlist control group were matched to the experimental group on age, grade, teacher, and diagnosis. None of the 16 participants were medicated for ADHD.

Results: Attention abilities as measured by the Test of Variables of Attention showed the experimental group of children with ADHD reduced errors of commission and anticipation, indicating a reduction in impulsivity. Teacher reports using the McCarney Scale indicated improvements in attention but no changes in impulsivity and hyperactivity.

Discussion: Several confounds require exploration before attribution of changes are assigned to neurofeedback. Whether the effects are due to the neurofeedback protocols, attendance at individual sessions away from the classroom, the attention of the technician, or the excitement of a special program cannot be determined with this study. It will be necessary to have a placebo group in order to separate systematically the variables in the training program.

KEYWORDS. Control group, McCarney Scale, neurofeedback, variables of attention

\section{INTRODUCTION}

Many reports have commented on the most frequent methods of treatment of Attention Deficit Hyperactivity Disorder (ADHD) over the past decade. The methods include medication (Copps, 1992; Silver, 1999; Spencer, Biederman \& Wilens, 2000; Wolraich, Lindgren, Stromquist, Milich, Davis \& Watson, 1990), behavior modification (Carlson \& Tamm, 2000; Klassen, Miller, Raina, Lee \& Olsen, 1999; Moss \& Dunlap, 1990; Rapport, Murphy \& Bailey, 1990), self-management techniques (Shapiro, DuPaul \& Bradley-Klug, 1998), and parent training programs (Barkley, 1998; Goldstein, 1997). Not all children respond favorably to medication with estimates that $30-40 \%$ of children showed no improvement or adverse effects (Green \& Barkley, 1996). Barkley (1997) has encouraged the addition of behavior modification programs in conjunction with medication to reduce the disruptive behaviors associated with ADHD. 
One behavior modification treatment option for ADHD is electroencephalographic (EEG) biofeedback treatment (Nash, 2000; Othmer, Othmer \& Kaiser, in press; Lubar \& Lubar, 1999). With EEG biofeedback, also known as neurofeedback, brainwaves of individuals are measured and the amplitudes of brainwaves are shown to the individuals receiving feedback. The intention of the technique is for the individual to alter the amplitudes of selected brainwaves. Efficacy of the treatment has been reported in case studies (Kaiser \& Othmer, 1998; Lubar \& Lubar, 1984; Lubar \& Shouse, 1976; Tansey \& Bruner, 1983) and group studies (Linden, Habib \& Radojevic, 1996; Lubar, Swartwood, Swartwood \& O'Donnell, 1995; Lubar, Swartwood, Swartwood \& Timmermann, 1995). Evaluations of the treatment have been conducted in laboratories (Lubar \& Shouse, 1976, 1977), clinics (Lubar \& Lubar, 1984; Lubar, Swartwood, Swartwood \& O’Donnell, 1995; Rossiter \& LaVaque, 1995; Tansey \& Bruner, 1983), and school settings (Boyd \& Campbell, 1998; Wadhwani, Radvanski, \& Carmody, 1998). After reviewing the nature of $\mathrm{ADHD}$, the public health concerns, and the results of QEEG studies of children with ADHD, Nash (2000) suggests there is a strong scientific rationale for neurotherapy as a treatment.

Outcomes of studies of groups of participants in clinical settings demonstrated academic changes attributed to EEG biofeedback training (Lubar \& Lubar, 1984; Lubar, Swartwood, Swartwood \& Timmermann, 1995). Training was designed to decrease theta activity either by inhibiting high-amplitude theta activity or by rewarding high-amplitude beta activity. For the participants who decreased their slow EEG activity, changes were found on a continuous performance task (TOVA). The investigators suggested that a reduction in theta activity is the key factor in modifying ADHD, especially for children below the age of fourteen. In another group study, EEG biofeedback training led to improvements in attention, behavior and intellectual functioning (Linden, Habib \& Radojevic, 1996). Wadhwani and others (1998) found neurofeedback training in a school setting to be related to improved scores on national achievement tests in a case study of a 10-year old boy on methylphenidate. The gains in performance were not sustained the year after training was completed. Boyd and Campbell (1998) reported improvements on the WISC-III Digit Span and the TOVA Inattention and Hyperactivity scales in five of six students receiving SMR training during twenty 30-minute training sessions conducted in a school environment. Several problems of operating in a school environment were reported including training, time commitment, and equipment issues.

The purpose of the current study was to assess the effectiveness of EEG biofeedback training in a controlled study conducted in an elementary school setting over a sustained time period. Effectiveness was assessed in three ways. First, the brainwaves of children were assessed for changes over the 
course of training. Second, classroom teachers monitored children for inattentive, impulsive, and hyperactive behaviors. Third, tests of variables of performance were assessed for changes over the several months of training.

This report briefly describes the results of implementation of a program designed to examine the effectiveness of neurofeedback conducted on site in an elementary school. The crossover study designed in the spring 1995 for implementation in the 1995-96 school year was intended to compare an experimental group to a waitlist control group that received treatment at a later date. Planned comparisons included intelligence test scores, national achievement test scores, patterns of brainwaves, tests of attention, school grades, attendance, and suspensions. Due to delays in the implementation of hardware, transfers of pupils to other school districts, and incomplete testing by school personnel, many of the educational variables were unavailable for evaluation on all participants at the end of the program in the summer 1996. The study does report on the effectiveness of neurofeedback on brainwaves, tests of attention, and scales of behavior.

Several decisions were made about the design in 1995 that may differ from decisions about current research practice. Hardware available to the program in 1995 was not artifact-free. Feedback protocols were based on recommendations of Lubar (1991) and Othmer (1993). The understanding of the various types of QEEG patterns in children with ADHD was not used to separate children into subgroups (Chabot \& Serfontein, 1996). We used a design that matched children with ADHD to children without ADHD on the variables of gender, age, and teacher for several reasons. Treatment was offered to parents and school personnel as a way of improving attention abilities in children. There was a preference that children not be highlighted socially in school by another program restricted to those diagnosed with ADHD. We wished to avoid the possibility that a diagnosis would be made on a child within the school in order to give them entry to the program. By offering the program to parents, we wished to enhance the image that the program was one of personal improvement and growth rather than another treatment required for their children.

\section{METHOD}

Participants. Sixteen elementary school children aged 8-10 years were selected to participate in the fall 1995 by the vice-principal of the school from a pool of fourth and fifth grade pupils. The ADHD-positive group consisted of eight participants identified by their teachers as having behavioral problems and diagnosed by a school psychologist as having ADHD (American Psychiatric Association, 1994). All pupils with ADHD were recommended to receive medication, however, their parents declined to utilize medication for 
their children. Participants were matched for age, gender, and grade in school with a group of eight students who were not diagnosed with attentional disorders. The protocol was reviewed and approved by the Board of Education of the school district. Parental consent was obtained for all participants after individual conferences were held with school administration and parents. In addition, the participants agreed to the study and were free to discontinue in the program when they wished. Participants were randomly assigned to the experimental or control groups as described in the design section. The experimental group was scheduled to receive training in the fall 1995 and the waitlist control group was scheduled to receive training in the spring 1996. Due to delays, the experimental group was trained in the spring 1996 and the control group was trained in the fall 1996.

The waitlist control group was added for several reasons. Our intention was for participants to be part of a special program at the school. By indicating to the children and their parents that the program was an attempt to help children improve their classroom performance and their behaviors at home, we intended to select students who were more likely to complete the training. In the event the program proved successful, we did not wish that children must be diagnosed with an attention disorder as a prerequisite to avail themselves of the program.

Setting. Participants were pupils at a public elementary school in Westchester County, New York. Neurofeedback sessions were conducted on site at the school in a room dedicated to training individual participants. Participants were released for 30 minutes from either standard classroom activities or recess for each session. Sessions were planned to be conducted in the morning three to four times weekly for each participant in the experimental group. Due to school assemblies and trips, scheduled days for standardized testing, holidays, storm days, and illness absences, the actual pattern of training sessions varied from the plan.

Trainer. A trainer from Biofeedback Consultants Incorporated served as the contact person for the pupil during sessions. The trainer had several responsibilities, starting with the technical tasks of placing electrodes accurately using a tape measure. Computer feedback programs were initiated, thresholds were set, and data were collected. The trainer coached participants by giving positive statements during the sessions. When the child reached a desired brainwave pattern, the trainer would say 'good job,' 'stay on task,' 'stay focused,' or 'good work.' Coaching the child into learning an awareness of when they produce increased beta or decreased theta is considered an essential aspect of training (Lubar \& Lubar, 1999). In parallel to the EEG training, the trainer performed several other tasks. The trainer observed the child during the training period and recorded notes regarding behavior, restlessness, and general attitude of student training. The children talked with the 
trainer about their classroom experiences. Brief consultations with the teachers were held on a daily or weekly basis to document teacher comments as to behavior of the children in classrooms. A supervisor from Biofeedback Consultants reviewed the reports weekly.

During the course of training, the trainer, the teachers, and school administrators monitored the classroom behaviors of the participants. At times, the classroom behaviors were observed and recorded for review. There were frequent discussions and conferences among the professionals to review progress in training and behaviors in the classroom. In the event the behaviors showed irritability, aggressive, or other signs of overstimulation, the electrode placement was changed from $\mathrm{C} 3$ to $\mathrm{Cz}$ and the frequency band to be enhanced was changed from $16-18 \mathrm{~Hz}$ to $13-15 \mathrm{~Hz}$.

Biofeedback Apparatus. EEG biofeedback equipment included hardware and software from the American Biotec Corporation. The unit was a CapScan Single Channel EEG/EMG, with a sample rate of 512 samples per second. The filter synthesis defined amplitude as the square root of sum of specified squared spectral bins from the FFT. Noise was input shortened at 0.7 RMS typical referred to input. The common mode rejection ratio was greater than $100 \mathrm{~dB}$ and the input impedance was greater than 100 meg-ohm shunted by 100 pf.

Hardware included three Grass Silver Cup Electrodes using monopolar electrode placement. An active electrode was placed at $\mathrm{C} 3$ or $\mathrm{Cz}$. A reference electrode would be placed on the same side as the active electrode. For example, during C3 training, the reference electrode was placed on the left ear and the ground electrode on the right ear. In CZ training, the reference electrode was placed on the right ear and the ground electrode on the left ear. Impedance was measured prior to each session and maintained at less than $10 \mathrm{~K}$ ohms to provide good electrode-skin contact. The amplified signals were sent to a 486 DX2/50 computer for visual and auditory feedback presentations displayed on a monitor to the participant.

Several of the participants initially showed high delta spiking. Therefore, the $2-7 \mathrm{~Hz}$ frequencies (delta-theta) were selected as the band to be suppressed while the $16-18 \mathrm{~Hz}$ frequencies (beta) were selected as the band to be reinforced for the protocol at the start of training.

Training Protocols. Auditory feedback was in the form of high-, medium-, and low-pitched tones representing respectively beta, EMG, and theta bands. Prior to each session, a threshold for beta was set so that on average beta activity reached or surpassed threshold 60-75 percent of the time. A threshold was set for EMG, activity in the $70-90 \mathrm{~Hz}$ frequencies range, so that on average EMG activity was less than threshold 60-75 percent of the time. A low-pitched tone would indicate when the pupil was below the threshold for delta-theta. The delta-theta threshold was adjusted at the start of each session 
so that on average theta activity was below threshold $60-75$ percent of the time. When reinforcement and inhibition task criteria were simultaneously met, the participant received auditory feedback tones and changes in the visual display on the monitor. When criteria were not simultaneously met, the feedback tones were muted and the visual display identified the frequency band that was out of preferred range. Amplitude data were collected continuously for the three bands over the 30-minute training session. Every three minutes, sample means for beta, EMG, and delta-theta were stored in an electronic file for subsequent analyses.

Alterations in Training Protocols. During the course of training, the participants in the ADHD-positive group showed signs of overstimulation within the first 13-35 sessions. For example, the child was more aggressive, expressed more anger, fought with peers or became agitated in the classroom. Information about overstimulation was accumulated from teachers, administrators, other staff (psychologists, social workers, classroom teacher aides) and the students themselves as observed during biofeedback by the trainer. This was an indication for a change in protocol. The 13-15 Hz SMR band replaced the $16-18 \mathrm{~Hz}$ beta band and the active electrode was changed from location $\mathrm{C} 3$ to $\mathrm{Cz}$. The thresholds were adjusted for SMR in the same manner as the adjustments and feedback reinforcement for beta. The participants in ADHD-positive group received beta protocols for the initial sessions (13 to 35 sessions varying among the children) and then were switched to the SMR protocol for the remaining sessions as indicated in Table 1. Participants in the ADHD-negative group received the beta protocol for the entire training period from January through May 1996.

McCarney Scale. McCarney developed the Attention Deficit Disorders Evaluation Scale (1989) to be completed by either teachers or paraprofessionals working directly with students during instructional situations. A total of 60 items describing specific behaviors were rated on a 5-point scale. Items were grouped into the dimensions of Inattentive (27 items), Impulsive (18 items), and Hyperactive (15 items). Raw scores were then standardized for each dimension and an overall Percentile Score was derived.

Performance Testing. The Test of Variables of Attention (TOVA) is a continuous performance test (CPT) designed to measure the visual attention style of participants (Greenberg, 1987; Greenberg \& Waldman, 1993). Testing was conducted with a PC desktop computer, a thumbswitch held in the preferred hand, and the TOVA program. Testing in one 22-minute session required participants to view a computer monitor and press a thumbswitch whenever a square figure appeared in the top portion of a rectangle. Participants were to inhibit the switch press when the square figure appeared in the lower half of the field. Dependent measures included reaction time, false presses, and missed events. However, the norms provided by the manufactur- 
TABLE 1. Participants

\begin{tabular}{|c|c|c|c|c|}
\hline $\begin{array}{l}\text { Group and Participant ID } \\
\text { Number of Sessions }\end{array}$ & Age & Gender & $\underline{\text { Grade }}$ & Diagnosis \\
\hline \multicolumn{5}{|l|}{ EXPERIMENTAL } \\
\hline A 48 & 10 & Female & 5 & ADHD-Positive \\
\hline B 47 & 9 & Male & 4 & ADHD-Negative \\
\hline C 36 & 9 & Male & 4 & ADHD-Positive \\
\hline D 48 & 9 & Male & 4 & ADHD-Positive \\
\hline E 40 & 9 & Male & 4 & ADHD-Negative \\
\hline F 40 & 9 & Male & 4 & ADHD-Negative \\
\hline G 40 & 10 & Female & 5 & ADHD-Negative \\
\hline $\mathrm{H} 44$ & 9 & Male & 4 & ADHD-Positive \\
\hline \multicolumn{5}{|l|}{ CONTROL } \\
\hline $\mathrm{CA}$ & 10 & Female & 5 & ADHD-Positive \\
\hline CB & 9 & Male & 4 & ADHD-Negative \\
\hline $\mathrm{CC}$ & 9 & Male & 4 & ADHD-Positive \\
\hline$C D$ & 9 & Male & 4 & ADHD-Positive \\
\hline $\mathrm{CE}$ & 9 & Male & 4 & ADHD-Negative \\
\hline $\mathrm{CF}$ & 9 & Male & 4 & ADHD-Negative \\
\hline CG & 10 & Female & 5 & ADHD-Negative \\
\hline $\mathrm{CH}$ & 9 & Male & 4 & ADHD-Positive \\
\hline
\end{tabular}

er for interpretation purposes were based on a standardization sample that differed from the participants in ethnicity. Therefore, the raw data rather than the standard scores were used to determine progress over the course of training.

Design. Each member of the eight matched pairs was randomly assigned to either the experimental or the control group. The experimental group consisted of four ADHD-positive and four ADHD-negative participants. Similarly, the control group consisted of four ADHD-positive and four ADHD-negative participants. Table 1 identifies the participants by age, gender, grade, and diagnosis for the experimental group member of each pair. Participants in the experimental group trained in individual sessions from January through May 1996. The control group was wait-listed. Classroom teachers completed the McCarney Scales for all 16 participants in January, March, and June 1996. The TOVA was administered individually to all 16 participants in January, March, and June 1996. The time between the January and June TOVA administrations ranged 142-163 days. The differences in treatment for the two 
groups were the EEG training sessions for the experimental group and no intervention for the control group. Teachers were aware when experimental group participants left the classroom to attend a biofeedback session.

Experimental Procedure. Experimental group participants trained in an office dedicated to feedback sessions. In the 30-minute sessions, participants sat in a chair in front of the computer monitor and a technician attached the electrodes on the head and ears. Participants viewed video games in which points were collected towards a reward of a display change. By altering levels of delta-theta and beta waves, participants learned to modify the audio and visual displays. Threshold levels were adjusted individually throughout the training sessions as participants made progress towards alterations of amplitudes. Sessions were conducted three to four times weekly for a total of 36 48 total sessions per participant. No other rewards were given for performance in terms of foods, free time from class, or higher grades. Participants were required to make up the classroom assignments they missed when attending training sessions. Control group participants did not leave the classroom for sham activities and did not participate in any activity to simulate interaction with a computer display.

All participants in both groups completed the TOVA in January, March, and June 1996. Teachers used the McCarney Scale to rate the classroom behaviors in the same months for all participants in both groups.

Scoring Procedures. Each session produced ten sample means for the three measures of beta, delta-theta, and EMG. Amplitudes of the frequency bands for the first five sessions were compared to the amplitudes of the last five sessions to determine if there were changes between the beginning and end of training.

The teacher ratings of Inattentive, Impulsive, and Hyperactive on the McCarney Scale were standardized for each dimension and an overall Percentile Score was derived. A series of analyses of variance were performed on the dependent measures of Inattention, Impulsivity, Hyperactivity, and Percentile Score to determine if there were changes over test administrations in the measures.

The raw scores, rather than the standardized scores, for the TOVA were used to compare groups for the following reasons. The TOVA was standardized for children on 775 children (377 boys, 398 girls) aged 6-16, randomly selected from classrooms in grades $1,3,5,7$, and 9. The standardization sample was mainly middle to upper-middle class, $99 \%$ Caucasian, from three suburban school districts in Minneapolis, Minnesota. Interestingly, children in special education classes were excluded from the sample (Greenberg \& Waldman, 1993, p. 1022). As suggested by the authors "First, one must keep in mind that the normative sample is not representative of most urban populations as it is heavily weighted toward middle- and upper-middle class and 
Caucasian subjects; hence, extreme caution must be exercised in applying these norms to lower-SES and non-Caucasian children. Nevertheless, the degree to which CPT assessments of inattention and impulsivity vary by SES and ethnicity remains an open, empirical issue" (Greenberg \& Waldman, 1993, p. 1029).

\section{RESULTS}

Brainwave Activity. A goal of training was to modify the amplitudes of the EEG in selected bandwidths, namely, to reduce amplitudes of delta-theta waves and increase the amplitudes of beta and SMR waves. One way to determine if the goals were met was to compare the amplitudes of brainwaves of early sessions to those in later sessions. EEG records were reviewed for the four participants in ADHD-positive subgroup (176 sessions, 88.0 hours) and for the four participants in the ADHD-negative subgroup (167 sessions, 83.5 hours).

Within each 30-minute session, there were 10 sample means representing three minutes of data collection of delta-theta and beta bands. By selecting the first five sessions as the baseline trials, there were 50 samples of amplitudes representing the first 2.5 hours of training for each pupil. The final five sessions yielded 50 samples representing the final 2.5 hours of training. By comparing the related samples, judgments of significant changes in amplitudes were made for each case. An example of the theta amplitudes for all sessions for one participant from the ADHD-positive group is shown in Figure 1 with amplitude on the vertical axis and sessions on the horizontal axis.

Note the variability and amplitude of the values of the three-minute samples. There were no significant changes in theta amplitude for this participant as assessed by regression analysis, $F(1,478)=0.146, p>.05$, slope $=-0.005$, $p>.05$. However, the protocol was changed from $\mathrm{C} 3$ beta to $\mathrm{Cz}$ SMR at session number 20 . Therefore, theta amplitudes were analyzed separately for each protocol as illustrated in Figure 2.

Amplitude values for all participants were compared using paired-t tests and Wilcoxon Signed Rank Tests. The rationale for both a parametric test and a nonparametric test was to ensure that the findings were not based on skewed data. For the delta-theta band, Table 2 shows the average amplitudes for initial and final sessions, test statistics, and the probabilities of significant changes.

Note there were no changes in electrode placement or reinforcement protocols for the four ADHD-negative pupils. Therefore, those comparisons are for 40-47 sessions of training. Three (Participants E, F, G) showed significant increases in delta-theta amplitudes and one (Participant B) showed no change. 
FIGURE 1. An example of the theta amplitudes in microvolts for all sessions for Participant D from the ADHD-positive group with amplitude on the vertical axis and sessions on the horizontal axis. Note the variability and amplitude of the values of the three-minute samples.

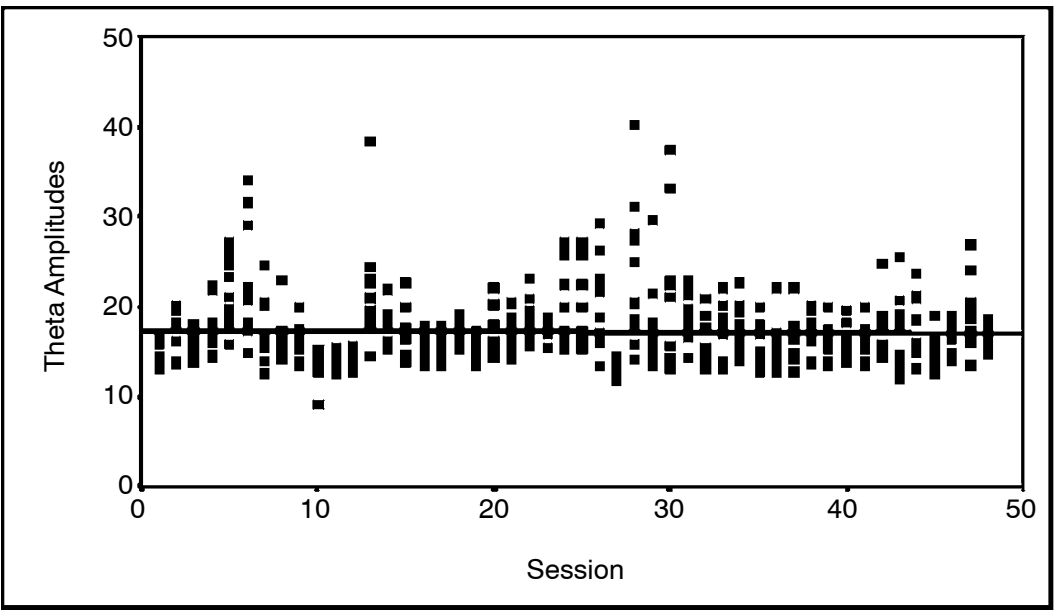

FIGURE 2. A comparison of the initial and final theta amplitudes in microvolts for Participant $D$ from the ADHD-positive group receiving neurofeedback. Note that the protocol changed from C3 beta to Cz SMR at session 20.

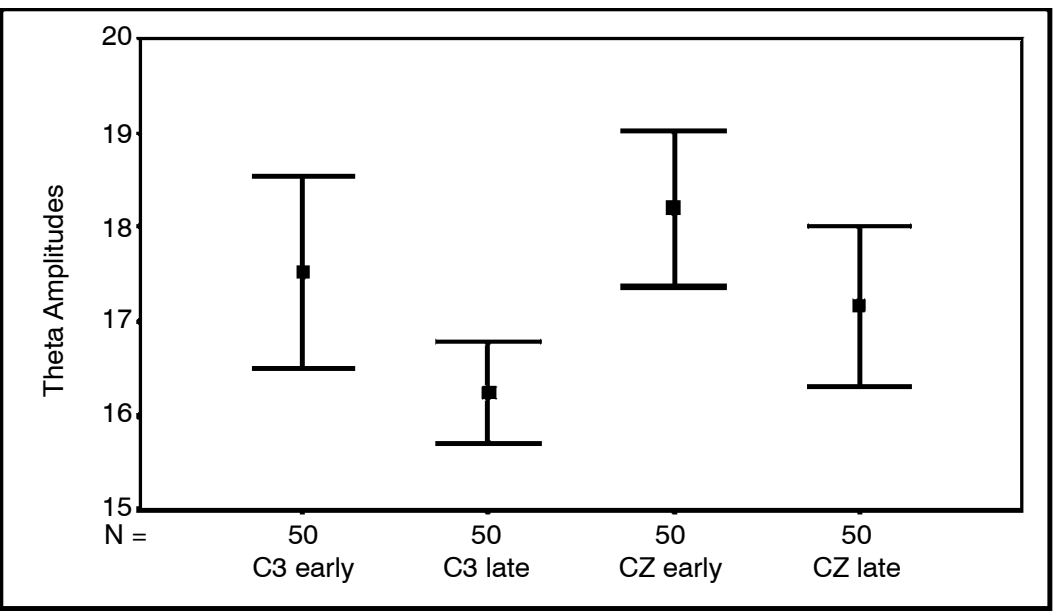


TABLE 2. Delta-Theta Changes Over Training

\begin{tabular}{|c|c|c|c|c|c|c|c|}
\hline $\begin{array}{l}\text { Participant } \\
\text { Group }\end{array}$ & $\begin{array}{c}\text { Number } \\
\text { Sessions } \\
\text { at } \\
\text { Location }\end{array}$ & $\begin{array}{l}\text { Early Sessions } \\
\text { Microvolts }\end{array}$ & $\begin{array}{l}\text { Later Sessions } \\
\text { Microvolts }\end{array}$ & $\begin{array}{c}\text { Paired } \\
\text { t-test }\end{array}$ & Prob & $\begin{array}{l}\text { Wilcoxon } \\
\text { Z test }\end{array}$ & Prob \\
\hline $\begin{array}{l}\text { Participant B } \\
\text { Control }\end{array}$ & $47 \mathrm{C} 3$ & 18.619 & 18.492 & .418 & $>.05$ & .164 & $>.05$ \\
\hline $\begin{array}{l}\text { Participant E } \\
\text { Control }\end{array}$ & $40 \mathrm{C} 3$ & 19.430 & 22.844 & 5.116 & .001 & 5.464 & .001 \\
\hline $\begin{array}{l}\text { Participant F } \\
\text { Control }\end{array}$ & $40 \mathrm{C} 3$ & 25.856 & 29.544 & 4.800 & .001 & 3.969 & .001 \\
\hline $\begin{array}{l}\text { Participant G } \\
\text { Control }\end{array}$ & $40 \mathrm{C} 3$ & 21.866 & 23.132 & 2.916 & .005 & 2.66 & .008 \\
\hline $\begin{array}{l}\text { Participant A } \\
\text { Experimental }\end{array}$ & $35 \mathrm{C} 3$ & 22.324 & 25.729 & 3.775 & .001 & 3.634 & .001 \\
\hline $\begin{array}{l}\text { Participant A } \\
\text { Experimental }\end{array}$ & $\begin{array}{l}13 \mathrm{Cz} \\
\text { SMR }\end{array}$ & 24.529 & 24.086 & .336 & $>.05$ & .767 & $>.05$ \\
\hline $\begin{array}{l}\text { Participant } \mathrm{C} \\
\text { Experimental }\end{array}$ & $13 \mathrm{C} 3$ & 21.878 & 21.785 & .090 & $>.05$ & .497 & $>.05$ \\
\hline $\begin{array}{l}\text { Participant C } \\
\text { Experimental }\end{array}$ & $\begin{array}{l}23 \mathrm{Cz} \\
\text { SMR }\end{array}$ & 20.926 & 21.557 & .682 & $>.05$ & 1.067 & $>.05$ \\
\hline $\begin{array}{l}\text { Participant D } \\
\text { Experimental }\end{array}$ & $19 \mathrm{C} 3$ & 17.518 & 16.253 & 2.224 & .031 & 1.950 & .051 \\
\hline $\begin{array}{l}\text { Participant D } \\
\text { Experimental }\end{array}$ & $\begin{array}{l}29 \mathrm{Cz} \\
\text { SMR }\end{array}$ & 18.203 & 17.160 & 1.898 & $>.05$ & 1.781 & $>.05$ \\
\hline $\begin{array}{l}\text { Participant } \mathrm{H} \\
\text { Experimental }\end{array}$ & $13 \mathrm{C} 3$ & 22.677 & 24.747 & 1.984 & .053 & 2.495 & .013 \\
\hline $\begin{array}{l}\text { Participant } \mathrm{H} \\
\text { Experimental }\end{array}$ & $\begin{array}{l}31 \mathrm{Cz} \\
\text { SMR }\end{array}$ & 20.814 & 21.503 & 1.207 & $>.05$ & 1.559 & $>.05$ \\
\hline
\end{tabular}

In contrast, analyses of the delta-theta amplitudes at training location $\mathrm{C} 3$ for the four ADHD-positive pupils showed different results. One pupil (Participant D) showed a significant decrease, one an increase (Participant $\mathrm{H}$ ), and two showed no change (Participants A, C). For the training at location $\mathrm{Cz}$, all four ADHD-positive children showed no change in delta-theta amplitudes. An analysis of the relationship of the initial with the final amplitudes for the eight $\mathrm{C} 3$ protocols and the four $\mathrm{Cz}$ protocols shows a significant correlation, $r(12)=0.912, p<0.01$. The change in amplitudes from initial to final sessions was positively, although not significantly correlated with the initial amplitudes, $r(12)=0.501, p=0.91$. The higher the initial delta-theta amplitudes, the more likely the change was an increase in the final amplitudes.

For the beta band training at location $\mathrm{C} 3$, Table 3 shows the average 
TABLE 3. Beta and SMR Changes Over Training

\begin{tabular}{|c|c|c|c|c|c|c|c|}
\hline $\begin{array}{l}\text { Participant } \\
\text { Group }\end{array}$ & $\begin{array}{c}\text { Number } \\
\text { Sessions } \\
\text { at } \\
\text { Location }\end{array}$ & $\begin{array}{l}\text { Early Sessions } \\
\text { Microvolts }\end{array}$ & $\begin{array}{l}\text { Later Sessions } \\
\text { Microvolts }\end{array}$ & $\begin{array}{c}\text { Paired } \\
\text { t-test }\end{array}$ & Prob & $\begin{array}{l}\text { Wilcoxon } \\
\text { Z test }\end{array}$ & Prob \\
\hline $\begin{array}{l}\text { Participant B } \\
\text { Control }\end{array}$ & $47 \mathrm{C} 3$ & 4.284 & 3.526 & 3.197 & .002 & 2.833 & .005 \\
\hline $\begin{array}{l}\text { Participant E } \\
\text { Control }\end{array}$ & $40 \mathrm{C} 3$ & 2.793 & 3.096 & 3.216 & .002 & 3.422 & .001 \\
\hline $\begin{array}{l}\text { Participant F } \\
\text { Control }\end{array}$ & $40 \mathrm{C} 3$ & 4.168 & 4.340 & 2.600 & .012 & 2.458 & .014 \\
\hline $\begin{array}{l}\text { Participant G } \\
\text { Control }\end{array}$ & $40 \mathrm{C} 3$ & 2.966 & 3.008 & .633 & $>.05$ & .340 & $>.05$ \\
\hline $\begin{array}{l}\text { Participant A } \\
\text { Experimental }\end{array}$ & $35 \mathrm{C} 3$ & 3.319 & 5.697 & 7.090 & .001 & 5.140 & .001 \\
\hline $\begin{array}{l}\text { Participant A } \\
\text { Experimental }\end{array}$ & $\begin{array}{l}13 \mathrm{Cz} \\
\text { SMR }\end{array}$ & 5.304 & 6.031 & 1.457 & $>.05$ & 1.738 & $>.05$ \\
\hline $\begin{array}{l}\text { Participant } \mathrm{C} \\
\text { Experimental }\end{array}$ & $13 \mathrm{C} 3$ & 4.404 & 5.267 & 1.802 & $>.05$ & 1.771 & $>.05$ \\
\hline $\begin{array}{l}\text { Participant } \mathrm{C} \\
\text { Experimental }\end{array}$ & $\begin{array}{l}23 \mathrm{Cz} \\
\mathrm{SMR}\end{array}$ & 5.025 & 3.907 & 3.312 & .002 & 3.007 & .003 \\
\hline $\begin{array}{l}\text { Participant } \mathrm{D} \\
\text { Experimental }\end{array}$ & 19 C3 & 4.135 & 3.514 & 2.180 & .034 & 1.689 & .091 \\
\hline $\begin{array}{l}\text { Participant D } \\
\text { Experimental }\end{array}$ & $\begin{array}{l}29 \mathrm{Cz} \\
\text { SMR }\end{array}$ & 4.636 & 4.510 & .383 & $>.05$ & .179 & $>.05$ \\
\hline $\begin{array}{l}\text { Participant } \mathrm{H} \\
\text { Experimental }\end{array}$ & $13 \mathrm{C} 3$ & 6.086 & 5.604 & 1.817 & $>.05$ & 1.815 & $>.05$ \\
\hline $\begin{array}{l}\text { Participant } \mathrm{H} \\
\text { Experimental }\end{array}$ & $\begin{array}{l}31 \mathrm{Cz} \\
\text { SMR }\end{array}$ & 5.291 & 6.160 & .045 & $>.05$ & 2.008 & .045 \\
\hline
\end{tabular}

amplitudes for initial and final sessions, test statistics, and the probabilities of significant changes. For the four ADHD-negative pupils, one (Participant B) showed a significant decrease in beta amplitudes, two (Participants E and F) showed significant increases and one evidenced no change (Participant G). In comparison, among the four ADHD-positive pupils, one (Participant A) showed a significant increase, one a decrease (Participant D), and two showed no changes in beta amplitudes (Participants $\mathrm{C}$ and $\mathrm{H}$ ).

For the SMR training at location $\mathrm{Cz}$, the four ADHD-positive pupils showed the following changes. One pupil (Participant $\mathrm{H}$ ) showed an increase, one a decrease (Participant C), and two (Participant A and D) showed no changes in SMR amplitudes. An analysis of the relationship of the initial and the final amplitudes for the eight $\mathrm{C} 3$ protocols and the four $\mathrm{Cz}$ protocols 
shows a significant correlation, $r(12)=0.624, p<.05$. The changes in amplitudes from initial to final sessions was negatively, although not significantly correlated with the initial amplitudes, $r(12)=-284, p>.05$. The higher the initial beta or SMR amplitudes, the more likely the change was a decrease in the final amplitudes.

McCarney Scale. Diagnostic groups were compared in a series of repeated-measures analyses of variance and non-parametric analyses for the dependent measures of Inattention, Impulsivity, Hyperactivity, and Percentile Score. $\mathrm{Eta}^{2}$ and omega ${ }^{2}$ are reported as estimates of the proportion of the total variability in the dependent variable that is accounted for by variation in the independent variable (Hayes, 1994).

Inattention scores improved significantly, $F(2,24)=3.624, p=.042$, eta ${ }^{2}=$ 0.232 , over the period from January $(M=5.94)$, through March $(M=6.31)$, to June $(M=7.38)$. Friedman non-parametric tests showed changes in the Experimental Group, Chi-square $(2)=12.250, p<.002$ but not the Control Group, Chi-square (2) $=1.040, p>.05$. To isolate the responsible levels of the factor, Wilcoxon Signed Ranks Tests showed no improvements in Inattention Scores from January to March, $z=1.890, p>.05$, but revealed significant improvements from March to June, $z=2.232, p<.03$.

No changes were found for Hyperactivity for the factors of Month or Group by means of analyses of variance. Analyses of Hyperactivity scores by means of Friedman non-parametric tests showed no changes in either the Experimental Group Chi-square (2) $=3.120, p>.05$ or the Control Group, Chi-square $(2)=1.615, p>.05$. Friedman non-parametric tests showed no changes in Impulsivity for either the Experimental Group Chi-square (2) = $0.667, p>.05$ or the Control Group, Chi-square $(2)=0.495, p>.05$.

A three way repeated measures analysis of variance on Percentile Scores revealed significant differences for groups, $F(1,12)=18.817, p<.001$, eta ${ }^{2}=$ .611 , and for test month, $F(2,24)=13.232, p<.001$, $e t a^{2}=.524$. As expected, the ADHD-negative group members $(M=48.63)$ were rated higher than the ADHD-positive participants $(M=7.00)$ in January. The June Percentile Scores $(M=41.50)$ were higher than both January $(M=27.81)$ and March Percentile Scores $(M=28.13)$. The interaction of Month and Group was reliable, $F(2,24)=3.582, p<.05$, eta $^{2}=.230$. Further analyses of Percentile Scores for each Group by repeated measures ANOVA isolated the causal factors. A significant interaction for Month and Diagnosis for the Experimental Group, $F(2,12)=15.452, p<.0005$, revealed that the ADHD-negative subgroup improved performance more than the ADHD-positive subgroup. Similar analyses for the Control Group showed no significant changes over time for either group. In summary, the Experimental Group showed changes in Percentile Scores and the Control Group showed no changes. Analyses by means of the Freidman nonparametric test showed increases in Percentile 
Scores for the Experimental Group, Chi-square $(2)=13.034, p<.001$ and no changes for the Control Group, Chi-square $(2)=1.500, p>.05$.

TOVA Performance. Repeated-measure analyses of variance and Friedman Tests were used to examine performance on the TOVA for the five measures of Commission Errors, Omission Errors, Anticipatory Scores, Reaction Time, and Variability of reaction time. Test Month was as a within-subject factor and Diagnostic Group and Treatment were between-subject factors.

There was no overall three-way interaction of Group, Diagnosis, and Month, $F(2,11)=0.184, p>.05$. Significant differences were found in Commission Errors over months, $F(2,24)=7.536, p=.003$, eta ${ }^{2}=.386$. No differences were found between groups or treatments. The experimental ADHD-positive group significantly decreased Commission Errors from January to June as assessed by regression analyses (slope $=-16.125, p<.05$; intercept $=66.333$ ) as illustrated in Figure 3; no other group had significant changes.

A series of paired t-tests comparing the months of January and June showed all participants in the experimental group decreased Commission Errors, $\mathrm{t}(7)=4.901, p=.002$, omega $a^{2}=.328$. The ADHD-positive group lowered errors significantly, $\mathrm{t}(3)=5.459, p<.01$, omega $a^{2}=.426$, one-tailed, as did the ADHD-negative group, $\mathrm{t}(3)=2.364, p<.05$, omega ${ }^{2}=.185$,

FIGURE 3. Means and 95\% Confidence Intervals for Commission Errors on the Test of Variables of Attention. The Experimental Group received EEG biofeedback training and the Control Group was waitlisted. The TOVA was administered in January, March and June 1996.

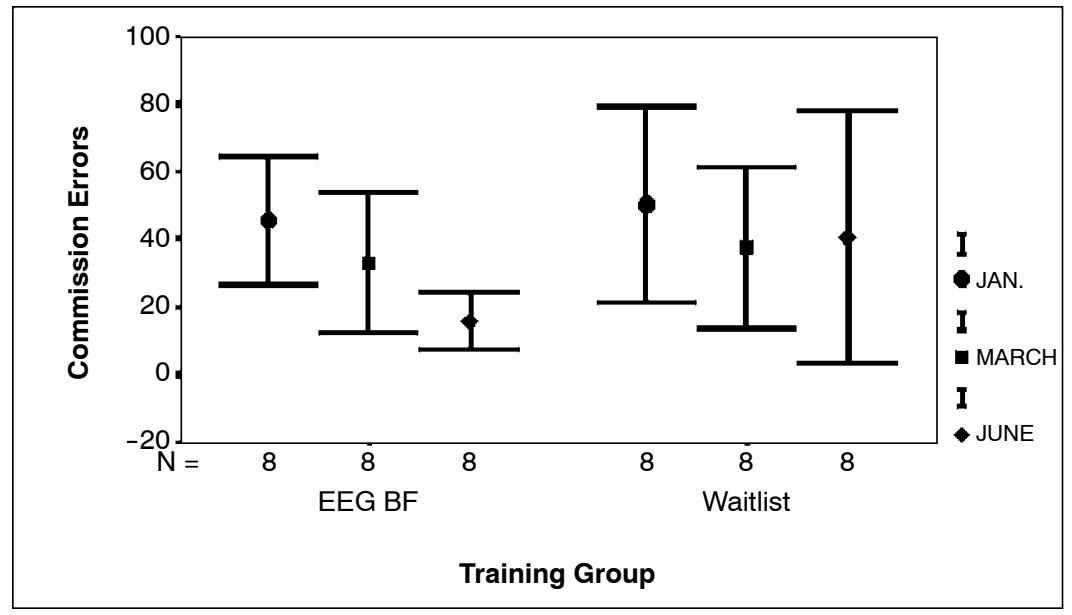


one-tailed. Nonparametric analyses did not confirm the changes in Commission Errors.

Although the Experimental Group started with much lower Anticipatory Scores than the Control Group, the experimental ADHD-positive group significantly decreased Anticipatory Scores as assessed by regression analyses (slope $=-3.000, p<.03$; intercept $=11.083$ ); no other group had significant changes in anticipatory scores. Nonparametric analyses did not confirm the changes in Commission Errors.

Significant differences in Omission Errors were found for the interaction of Diagnostic Group and Month, $F(2,24)=3.579, p<.05 ;$ eta $^{2}=.230$. Participants in the ADHD-positive group had lower Omission Errors in June than all other months and groups. Based on an ANOVA of the ADHD group, there were no differences between groups, $F(2,12)=0.776, p>.05$, eta ${ }^{2}=$ .114. Nonparametric Freidman Tests found no changes for the ADHD-negative participants, $p>.05$, but did reveal reductions in Omissions Errors for both Experimental ADHD-positive, Chi-square $(2)=6.500, p<.04$, and Control ADHD-negative participants, Chi-square $(2)=6.500, p<.04$.

No differences were found in Response Time, or Variability Scores for the factors of Group, Month, Diagnosis, or any interaction of the variables by repeated measures ANOVA, regression analyses, or nonparametric tests (all $p>$.05).

\section{DISCUSSION}

All eight participants completed the course of biofeedback training, ranging from 36 to 48 sessions. This finding demonstrates the persistence and motivation of elementary school children to sustain an intervention in the absence of external rewards. As suggested by Green and Barkley (1996), it is necessary to focus on intervention strategies within traditional settings such as school in order to achieve the desired generalization of behaviors. Based on teacher ratings, there were no changes in the impulsive or hyperactivity behaviors of children in either the Experimental or Control Groups. The only scale showing improvement based on teacher ratings was inattention. Many of the teachers at the setting of the study voiced doubts about the effectiveness of any behavioral treatment on the behavior of the ADHD participants. The requirement of completing the McCarney Scale forms was an additional burden to the classroom teachers. Explanations for the lack of change in behaviors assessed by teachers may be found in a case study of the effects of placebo interventions on teacher ratings of student behaviors. In a study of the effects of methylphenidate on classroom behavior, Hyman and colleagues have reported that teacher ratings on ADHD scales are susceptible to placebo effects (Hyman, Wojtowicz, Lee, Haffner, Fiorello, Storlazzi \& Rosenfeld, 
1998). Others have suggested that teacher ratings are not sensitive to the changes in behavior shown by children to parents (Klassen, Miller, Raina, Lee \& Olsen, 1999).

In contrast to the teacher ratings, changes in attention abilities were evident in performance in the TOVA task. In summary, based on the TOVA measures of Commission Errors and Anticipatory Scores, there was a genuine reduction in hyperactivity as evidenced by fewer false actions for the ADHD-positive group receiving EEG biofeedback compared to other groups. There were no changes in the Control Group. The changes cannot be attributed to maturation, time of year, or experience with the test because the groups were matched for age, grade, and diagnosis and tested in the same three months. All participants had the same level of experience with the performance testing conditions. None of the participants were on medication; therefore the changes in performance cannot be attributed to medication.

Changes in brainwave activity over the course of training were not uniform over all participants. For the amplitudes of delta-theta activity among the participants with ADHD, one demonstrated a decrease, one an increase, and two showed no changes in amplitudes. For the participants without ADHD, three showed increases in amplitudes and one showed no change. These findings are not in agreement with those reported in the literature about reductions in theta activity with neurofeedback training. Perhaps the inclusion of the delta band $(2-3 \mathrm{~Hz})$ within the low frequency channel (typically 4-7 Hz) led to changes that are different from the reductions in theta amplitudes reported after 40 sessions of treatment (Lubar, Swartwood, Swartwood \& O'Donnell, 1995). Another viewpoint on theta changes can be found in the work of Ramos (1998) who reported successful application of neurofeedback when the theta band was rewarded along with beta.

For beta range activity among the ADHD-negative participants, two showed increases, one a decrease, and one showed no change in beta amplitudes. Among the ADHD-positive participants, one increased, one decreased, and two showed no change in amplitudes in beta. For the SMR training, one pupil showed an increase, one a decrease and two no changes in amplitudes. In summary, there were no changes in brainwaves in beta or SMR as expected based on the recommendations for protocols in the literature. It may be that the value of biofeedback is to provide a steady level of relaxed attention, achieved either by reductions in excessive beta activity or by increases in initial beta levels (Lubar \& Shouse, 1976, 1977; Linden, Habib \& Radojevic, 1996).

The lack of changes in brainwaves in a study designed to demonstrate that the feedback of brainwaves is instrumental in behavioral change requires explanation. For example, Furedy (1987) indicated the essential evidence is a change in the physiological mechanism receiving feedback. In this study, the 
changes in EEG would be required to demonstrate a relationship to behavioral changes. These issues are addressed by Lubar and Lubar (1999) and by Othmer, Othmer and Kaiser (1999). Findings on the relationship of EEG changes in children taking methylphenidate have shown reductions in theta and alpha with increases in beta only for children who had a positive medication response (Loo, Teale \& Reite, 1999).

In general, major changes in brainwaves were noted after 20-25 sessions of training. This finding suggests that short-term training ( 20 sessions or less) of EEG biofeedback may not be sufficient for changes in behavior to be noted. In review of the teacher ratings, the percentile scores showed no changes from the January to the March sample periods. However, there were significant improvements in Percentile Scores for the Experimental Group from March to June corresponding to the training sessions beyond the first 20-25 sessions. There were no changes in Percentile Scores for the wait-list control group over the same six-month period from January to June. There were confounds in the design due to changes in protocols from beta to SMR for the ADHD-positive group. Beta protocols are generally used to promote alertness in individuals who show lethargy or depression. The SMR protocols are used generally to reduce agitation and promote relaxed attention. During training, several participants became agitated under the beta training protocol and exhibited more hyperactivity as noted by teachers. When the protocols were changed to SMR, hyperactivity was reduced. As shown in Table 3, protocols were changed for the four ADHD participants from beta to SMR after 13-35 sessions. The protocol remained as beta for the four participants in the ADHD-negative group.

There were some experiences common to participants in both the experimental and control groups. The 16 children were selected to participate in a special program from among the 1000 pupils at the school. All participants received increased attention by the technicians administering the TOVA tests and by the teachers who completed the McCarney Scales on three occasions. However, there are confounds in the design that will require investigation. The experimental group had several experiences not available to the control group. The eight children in the experimental group had individual time with a technician in an isolated room, received EEG biofeedback and were rewarded with changes in a video game when brainwave activity was maintained within specified amplitude ranges. The comparison control group had no contact with the training technicians, did not have 30-minute sessions in an isolated room, and engaged in no activity providing them with immediate feedback. Therefore, it will be necessary to investigate the relative contributions of the several variables on changes in test performance. The four major variables are the presence of an individual trainer, training in a room separate from the classroom, changes in a video game, and the effect of controlling the 
game with brainwave changes. It would be necessary to conduct a study where the four variables are isolated to determine if the brainwave control is the sufficient cause of behavioral change.

A second source of variability was the selection of participants. The effects of comorbid diagnoses may affect the EEG sufficiently to mask differences between ADHD and non-ADHD groups. Estimates run as high as more than 50\% co-morbidity of ADHD with other disorders such as anxiety, depression, and conduct disorders (Biederman, Newcorn \& Sprich, 1991; Spencer, Biederman \& Wilens, 1999). Many of the behaviors such as poor social skills, problems at home with parents, and low academic functioning may be caused by other disorders. For example, mania is comorbid with ADHD (Carlson, 1998).

As discussed by Hodes and Woodard (1997), there is considerable scrutiny of the use of EEG biofeedback for the treatment of ADHD. Many studies have reported favorable results with neurofeedback. However, a placebo control study would allow separation of the many variables entering the treatment session such as the individualized attention the child receives, the novelty of the setting, and the freedom from school distractions typically absent in laboratory and clinical settings. Several research groups have indicated the need for controlled studies with large populations (Lubar \& Lubar, 1999; Othmer, Othmer, \& Kaiser, 1999).

There were several new contributions of the effectiveness of EEG biofeedback in the remediation of behavioral problems. The study was conducted on site in a school setting with the distractions found in many elementary schools, and the participants remained in the school environment without requiring transportation to a clinical or laboratory setting. Throughout the five months, the Control Group children attended the same classes with the same teachers and participated in the same activities as the Experimental Group children with the exception of the biofeedback sessions.

In terms of the acceptance of the program by the school administration, teachers, parents and pupils, there were favorable responses. Pupils in other classes asked to be part of the continuation of the program, and parents requested that their children be considered for the next year. Teachers reported that children smiled more, were more attentive to assignments, and interacted more appropriately in class. Many of the statements and descriptions did not show as data in the McCarney Scale or in the TOVA testing. It may be necessary to develop questionnaires that document such behaviors to be used at the start of each academic year to determine how many of the pupils make such changes without participation in the training program. Similar projects were implemented in other elementary schools in the same district. A descriptive report of the reactions was documented in the book, $A$ Symphony in the Brain (Robbins, 2000). 


\section{CONCLUSIONS}

In this study, it was demonstrated that individuals diagnosed clinically by maladaptive behaviors showed genuine changes in impulsivity and hyperactivity as a result of participation in the EEG biofeedback program. Whether the effects are due to the specific protocols, attendance at sessions, the excitement of special attention, or the technician cannot be determined at this time. It will be necessary to have a placebo group in order to separate systematically the variables in the training program. The findings of this study with non-medicated children cannot be generalized to children with ADHD who are on psychotropic medications to reduce hyperactive symptoms.

\section{ACKNOWLEDGMENTS}

Portions of this research were supported by a Faculty-Student research grant awarded by Saint Peter's College. The authors wish to thank Universal Attention Disorders, Inc. and The TOVA Research Foundation of St. Paul, Minnesota for generously donating TOVA tests for this study. Details concerning the biofeedback training can be obtained from Dr. Mary Jo Sabo, Biofeedback Consultants Incorporated, 21 South Amundsen Lane, Suffern, NY 10901 (E-mail: SABOEEG@AOL.COM), <www.TheRippleEffect.Org> Details concerning the social and educational impact of the training can be obtained from Linda Vergara, Principal, Enrico Fermi School for the Performing Arts, 27 Poplar Street, Yonkers, NY 10701.

\section{REFERENCES}

American Psychiatric Association. (1994). Diagnostic and statistical manual IV. (DSM-IV). Washington, DC: Author.

Barkley, R. A. (1998). Attention-deficit hyperactivity disorder: A handbook for diagnosis and treatment. New York: Guilford. Second edition.

Barkley, R. A. (1997). ADHD and the nature of self-control. New York: Guilford.

Biederman, J, Newcorn, J., \& Sprich, S. (1991). Comorbidity of attention deficit hyperactivity disorder with conduct, depressive, anxiety, and other disorders. American Journal of Psychiatry, 148, 564-577.

Boyd, W. D., \& Campbell, S. E. (1998). EEG biofeedback in the schools: The use of EEG biofeedback to treat ADHD in a school setting. Journal of Neurotherapy, 2(4), 65-71.

Carlson, G. A. (1998). Mania and ADHD: Comorbidity or confusion. Journal of Affective Disorders, 51(2), 177-187.

Carlson, C. L., \& Tamm, L. (2000). Responsiveness of children with attention deficit-hyperactivity disorder to reward and response cost: Differential impact on performance and motivation. Journal of Consulting and Clinical Psychology, 68(1), 73-83. 
Chabot R. J., \& Serfontein, G. (1996). Quantitative electroencephalographic profiles of children with attention deficit disorder. Biological Psychiatry, 40, 951-963.

Copps, S. C. (1992). The attending physician: Attention deficit disorder. Atlanta: SPI Press.

Furedy, J. J. (1987). Specific versus placebo effects in biofeedback training: A critical lay perspective. Biofeedback and Self-Regulation, 12, 169-184.

Goldstein, S. (1997). Managing attention and learning disorders in late adolescence and childhood: A guide for practitioners. New York: Wiley.

Greene, R. W., \& Barkley, R. A. (1996). Attention deficit hyperactivity disorder: Diagnostic, developmental, and conceptual Issues. In M. J. Breen and C. R. Fiedler (Eds.) Behavioral approach to assessment of youth with emotional/behavioral disorders. Austin: Pro-Ed.

Greenberg, L. M. (1987). An objective measure of methylphenidate response: Clinical use of the MCA. Psychopharmacology Bulletin, 23(2), 279-282.

Greenberg, L. M., \& Waldman, I. D. (1993). Developmental normative data on the Test of Variables of Attention (T.O.V.A.). Journal of Child Psychology \& Psychiatry \& Allied Disciplines, 34(6), 1019-1030.

Hayes, W. L. (1994). Statistics. New York: Harcourt Brace College Publishers.

Hodes, R. L., \& Woodard, A. R. (1997). The biofeedback treatment of neurological and neuropsychological disorders of childhood and adolescents. In C. R. Reynolds and E. Fletcher-Janzen (Eds.) Handbook of clinical child neuropsychology (pp. 418-438). New York: Plenum.

Hyman, I. A., Wojtowicz, A., Lee, K. D., Haffner, M. E., Fiorello, C. A., Storlazzi, J. J., \& Rosenfeld, J. (1998). School-based methylphenidate placebo protocols: Methodological and practical issues. Journal of Learning Disabilities, 31(6), 581-594, 614.

Kaiser, D. A., \& Othmer, S. (1998). Efficacy of SMR-beta neurofeedback on attentional processes. T.O.V.A. News, 4(2), 3-5.

Klassen, A., Miller, A., Raina, P., Lee, S. K., Olsen, L. (1999). Attention-deficit hyperactivity disorder in children and youth: A quantitative systematic review of the efficacy of different management strategies. Canadian Journal of Psychiatry, 44(10), 1007-1016.

Linden, M., Habib, T., \& Radojevic, V. (1996). A controlled study of the effects of EEG biofeedback on cognition and behavior of children with Attention Deficit Disorder and learning disabilities. Biofeedback and Self-Regulation, 21, 35-48.

Loo, S. K., Teale, P. D., Reite, M. L. (1999). EEG correlates of methylphenidate response among children with ADHD: A preliminary report. Biological Psychiatry, 45(12), 1657-1660.

Lubar, J. F. (1991). Discourse on the development of EEG diagnostics and biofeedback for attention-deficit/hyperactivity disorders. Biofeedback and Self-Regulation, 16, 201-225.

Lubar, J. O., \& Lubar, J. F. (1984). Electroencephalographic biofeedback of SMR and beta for treatment of attention deficit disorders in a clinical setting. Biofeedback and Self-Regulation, 9, 1-23.

Lubar, J. F., \& Lubar, J. O. (1999). Neurofeedback assessment and treatment for attention-deficit/hyperactivity disorders. In J. R. Evans and A. Abarbanel (Eds.) 
Introduction to quantitative EEG and neurofeedback (pp. 103-143). New York: Academic Press.

Lubar, J. F., \& Shouse, M. N. (1976). EEG and behavioral changes in a hyperkinetic child concurrent with training of the sensorimotor rhythm (SMR): A preliminary report. Biofeedback and Self-Regulation, 1, 293-305.

Lubar, J. F., \& Shouse, M. N. (1977). Use of biofeedback in the treatment of seizure disorders and hyperactivity. Advances in Child Clinical Psychology, 1, 204-251.

Lubar, J. F., Swartwood, M. O., Swartwood, J. N., \& Timmermann, D. L. (1995). Quantitative EEG and auditory event-related potentials in the evaluation of attention-deficit/hyperactivity disorder: Effects of methylphenidate and implications for neurofeedback training. Journal of Psychoeducational Assessment, ADHD Special, 143-160.

Lubar, J. F., Swartwood, M. O., Swartwood, J. N., \& O’Donnell, P. H. (1995). Evaluation of the effectiveness of EEG neurofeedback training for ADHD in a clinical setting as measured by changes in T.O.V.A. scores, behavioral ratings, and WISCR performance. Biofeedback and Self-Regulation, 20, 83-99.

McCarney, S. B. (1989). Attention deficit disorders evaluation scale. Colombia: Hawthorne Educational Services.

Moss, R. A., \& Dunlap, H. H. (1990). Why Johnny can't concentrate: Coping with attention deficit problems. New York: Bantam Books.

Nash, J. K. (2000). Treatment of attention deficit-hyperactivity disorder with neurotherapy. Clinical Electroencephalography, 31 (1), 30-37.

Othmer, S. (1993). EEG Spectrum training syllabus, Volume two. Encino: EEG Spectrum.

Othmer, S., Othmer, S. F., \& Kaiser, D. A. (1999). EEG biofeedback: An emerging model for its global efficacy. In J. R. Evans and A. Abarbanel (Eds.) Introduction to quantitative EEG and neurofeedback (pp. 243-310). New York: Academic Press.

Othmer, S., Othmer, S. F., \& Kaiser, D. A. (in press). EEG biofeedback: A generalized approach to neuroregulation. In R. Kall, J. Kamiya, and G. Schwartz (Eds.) Applied neurophysiology \& brain biofeedback.

Ramos, F. (1998). Frequency band interaction in ADD/ADHD neurotherapy. Journal of Neurotherapy, 3(1), 26-41.

Rapport, M., Murphy, A., \& Bailey, J. S. (1990). The effects of a response cost treatment tactic on hyperactive children. Journal of School Psychology, 18, 98.

Robbins, J. (2000). A symphony in the brain. New York: Atlantic Monthly Press.

Rossiter, T. R., \& LaVaque, T. J. (1995). A comparison of EEG biofeedback and psychostimulants in treating attention deficit hyperactivity disorders. Journal of Neurotherapy, 1(1), 48-59.

Shapiro, E. S., DuPaul, G. J., \& Bradley-Klug, K. L. (1998). Self-management as a strategy to improve the classroom behavior of adolescents with ADHD. Journal of Learning Disabilities, 31, 545-555.

Silver, L. B. (1999). Alternative (nonstimulant) medications in the treatment of attention-deficit/hyperactivity disorder in children. Pediatric Clinics of North America, 46(5), 965-975. 
Spencer, T., Biederman, J., \& Wilens, T. (1999). Attention-deficit/hyperactivity disorder and comorbidity. Pediatric Clinics of North America; 46(5), 915-927.

Spencer, T., Biederman, J., \& Wilens, T. (2000). Pharmacotherapy of attention deficit hyperactivity disorder. Childhood and Adolescent Psychiatric Clinics of North America, 9(1), 77-97.

Tansey, M. A., \& Bruner, R. L. (1983). EMG and EEG biofeedback training in the treatment of a 10-year-old hyperactive boy with a developmental reading disorder. Biofeedback and Self-Regulation, 4, 299-311.

Wadhwani, S., Radvanski, D. C., \& Carmody, D. P. (1998). Neurofeedback training in a case of attention deficit hyperactivity disorder. Journal of Neurotherapy, 3(1), 42-49.

Wolraich, M. L., Lindgren, S., Stromquist, A., Milich, R., Davis, C., \& Watson, D. (1990). Stimulant medication use by primary care physicians in the treatment of attention-deficit hyperactivity disorder. Pediatrics, 86, 95-101.

RECEIVED: 02/01/00

REVISED: 06/28/00

ACCEPTED: 07/15/00

\section{HAWORTH JOURNALS} ARE AVAILABLE ON MICROFORM

All Haworth journals are now available in either microfiche or microfilm from The Haworth Microform/Microfiche Division at the lowest possible prices.

Microfiche and microfilms are available at $25 \%$ above the "library" subscription rate. For journal subscription rates, please look within the journal on the copyright pages.

For all microform subscriptions, these charges apply: outside US and Canada: $40 \%$ to total; in Canada, $30 \%$ to total as well as $7 \%$ GST.

Microfilm specifications: $35 \mathrm{~mm}$; diazo or silver.

Microfiche specifications: $105 \mathrm{~mm} \times 184 \mathrm{~mm}$ (4" x 6"); reduction ratio: $24 \mathrm{X}$; nonsilver (diazo) positive polarity.

Microform are mailed upon completion of each volume.

For further information, contact Janette Kemmerer, Microform Contact, The Haworth Press, Inc., 10 Alice Street, Binghamton, NY 13904-1580; Tel: (607) 722-5857, ext. 311; Fax: (607) 722-1424;

E-Mail: getinfo@haworthpressinc.com

Microform and microfiche are also available from Bell \& Howell Information and Learning (formerly University Microfilms International), 300 North Zeeb Road, Ann Arbor, MI 48106-1346; Tel: (800) 521-0600. 